\title{
MANY-ELECTRON RELATIVISTIC CALCULATION AND INTERPRETATION OF ATOMIC PROCESSES IN TIME DEPENDENT HEAVY-ION SCATTERING
}

\author{
B. THIES, W.-D. SEPP and B. FRICKE \\ Department of Physics, University of Kassel, D-3500 Kassel, FRG
}

Received 24 May 1989; accepted for publication 9 June 1989

Communicated by J.P. Vigier

\begin{abstract}
The time dependence of a heavy-ion-atom collision system is solved via a set of coupled channel equations using energy eigenvalues and matrix elements from a self-consistent field relativistic molecular many-electron Dirac-Fock-Slater calculation. Within this independent particle model we give a full many-particle interpretation by performing a small number of single-particle calculations. First results for the $P(b)$ curves for the Ne K-hole excitation for the systems $\mathrm{F}^{8+}-\mathrm{Ne}$ and $\mathrm{F}^{6+}-\mathrm{Ne}$ as examples are discussed.
\end{abstract}

The time dependence of a general time dependent quantum mechanical many-electron system is correctly described by solving the time dependent Dirac equation. The best approximation which might be feasible for the description of a many-electron ionatom collision is the time dependent Hartree(Dirac-)Fock equation but its solution is still not possible in practice. We instead try to solve the time dependent Dirac equation by approximating the Hamiltonian by a sum of adiabatic one-particle Dirac-Fock Hamiltonians. Expanding the wavefunction in realistic molecular many-electron DiracFock wavefunctions leads then to a set of coupled channel equations. With this method we are able to give a self-consistent many-electron relativistic time dependent description in complicated ion-atom collisions for all internuclear distances. We present this Letter because such an accurate approach has never been performed before. As a first example we have decided to take up the case $\mathrm{F}^{8+}$ and $\mathrm{F}^{6+}$ on $\mathrm{Ne}$ for different impact energies because this system has been measured very accurately [1] although (of course) the relativistic effects are negligible here. But the method has the great advantage that it can directly be used for heavier systems. An overview of the various approximations used so far can be found in ref. [2]. For the specific case of $\mathrm{F}^{8+}$ on $\mathrm{Ne}$ an $\mathrm{AO}^{+}$ calculation by Fritsch and Lin [3] and an effective potential treatment by Töpfer et al. [4] exist.

We define time dependent single-particle collision states $\left|\psi_{n}(t)\right\rangle$ as the solutions of the single-particle time dependent Dirac equation

$\bar{h}^{\text {eff }}(\boldsymbol{R}(t))\left|\psi_{n}(t)\right\rangle=\mathrm{i} \hbar \frac{\mathrm{d}}{\mathrm{d} t}\left|\psi_{n}(t)^{\prime}\right\rangle$,

where $h^{\text {eff }}$ is defined as the single-particle HartreeFock Hamiltonian.

To solve eq. (1) we expand the single-particle scattering wavefunctions $\left|\psi_{n}(t)\right\rangle$ in a (complete) set of single-particle basis states,

$\left|\psi_{n}(t)\right\rangle=\sum_{k}\left|\phi_{k}(\boldsymbol{R}(t))\right\rangle c_{k n}(t)$.

For $\left|\phi_{k}(t)\right\rangle$ we use the single-particle wavefunctions from static diatomic self-consistent relativistic DiracFock-Slater calculations [5] as a basis. Inserting this into eq. (1) gives the equivalent single-particle matrix (coupled channel) equations

$\mathrm{i} \hbar \mathbf{s} \frac{\mathrm{d}}{\mathrm{d} t} \boldsymbol{c}_{n}=\mathbf{m} \boldsymbol{c}_{n}, \quad n=1,2, \ldots, N$,

for the column vectors $c_{n}=\left(c_{k n}\right) . \mathbf{s}=\left(s_{l k}\right)$ is the overlap matrix and $\mathbf{m}=\left(m_{l k}\right)$ the coupling matrix. Eigenvalues and radial as well as rotational coupling 
matrix elements are taken from the static calculations [5] for a large number of internuclear distances. As result of such coupled channel calculations we get a set $\left|\psi_{n}(t)\right\rangle$ of solutions of eq. (1) to $N$ mutual orthogonal initial conditions. It has been shown [6] that just one Slater determinant $|\Psi(t)\rangle$ which is built up from $N$ single-particle functions $\left|\psi_{n}(t)\right\rangle$ is a solution of the many-particle time dependent Dirac equation

$\hat{H}^{\mathrm{eff}}(\boldsymbol{R}(t))|\Psi(t)\rangle=\mathrm{i} \hbar \frac{\mathrm{d}}{\mathrm{d} t}|\Psi(t)\rangle$,

where the exact Hamiltonian $\hat{H}_{\mathrm{e}}$ is replaced by the effective Hamiltonian $\hat{H}^{\text {eff }}$,

$\hat{H}^{\mathrm{eff}}(\boldsymbol{R})=\sum_{i=1}^{n} \hat{h}_{i}^{\mathrm{eff}}(\boldsymbol{R})$

A formal solution of eq. (4) could be achieved by expanding the total scattering wavefunction in a complete set of time dependent many-electron (configuration space) wavefunctions $\left|\Phi_{K}(t)\right\rangle$ where each many-electron wavefunction $\left|\Phi_{K}(t)\right\rangle$ is built up from $N$ single-particle basis functions $\left|\phi_{k}(t)\right\rangle$,

$$
|\Psi(t)\rangle=\sum_{K}\left|\Phi_{K}(t)\right\rangle C_{K}(t)
$$

which leads to a set of coupled channel equations for $C$ analogous to eq. (3).

Both sets of $\mathbf{c}$ and $C$ are absolutely equivalent and contain the same physical information. The connection between both sets has first been discussed in ref. [6]. For each state $n$ in which an electron is present a separate coupled channel calculation has to be performed. As result one gets $N$ sets of amplitudes $c_{k n}$ where $k$ marks the final state. These $c_{k n}$ are then used for the interpretation in the many-particle picture, i.e. the $C_{k}$ from eq. (6). These amplitudes now allow us to answer different questions. For example one may ask how large is the chance of finding one hole or two holes or at least one hole in a certain atomic level.

As first examples we are interested in understanding the systems $\mathrm{F}^{8+}-\mathrm{Ne}$ and $\mathrm{F}^{6+}-\mathrm{Ne}$ because a large number of experimental results are available [1]. The experiment is performed with the last question how large the chance is of finding at least one hole in the Ne $1 \mathrm{~s}$ shell in the outgoing scattering systems. These

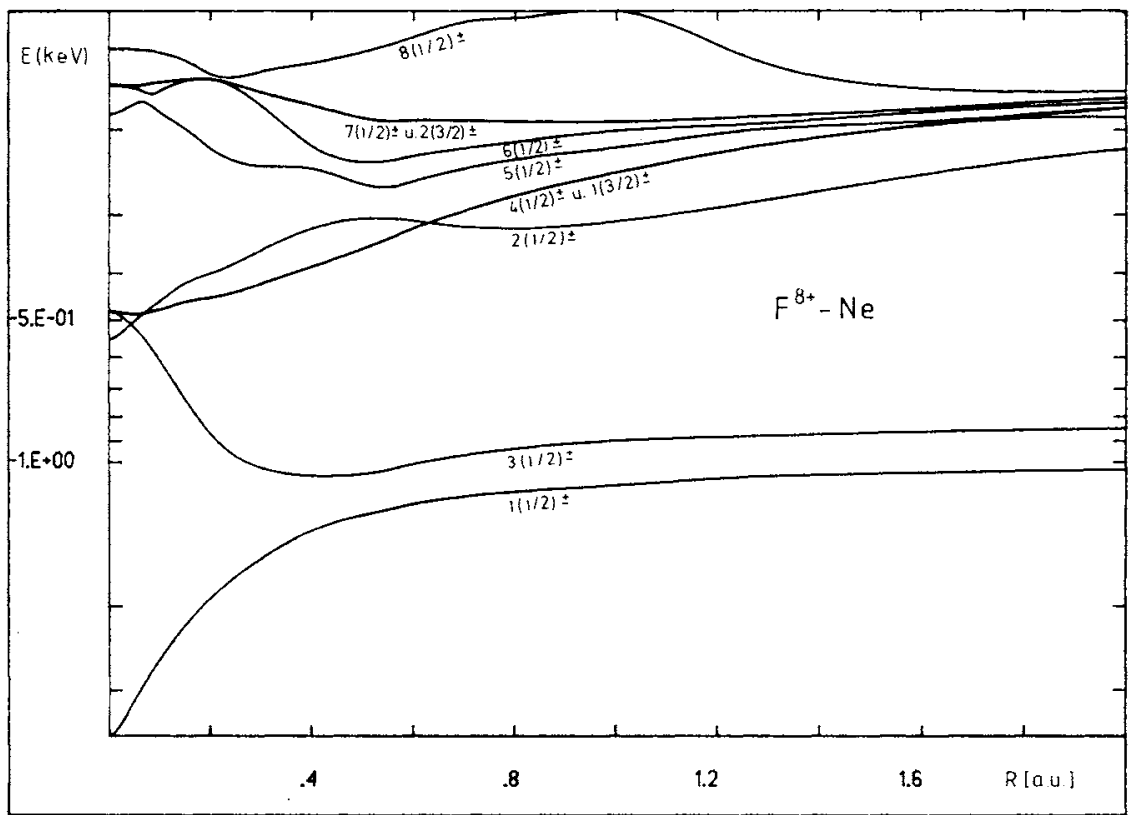

Fig. 1. Correlation diagram for the system $F^{8+}-\mathrm{Ne}$. (Due to diabatization the second level is called $3(1 / 2)$ because it correlates with the third level at $R=0$.) 

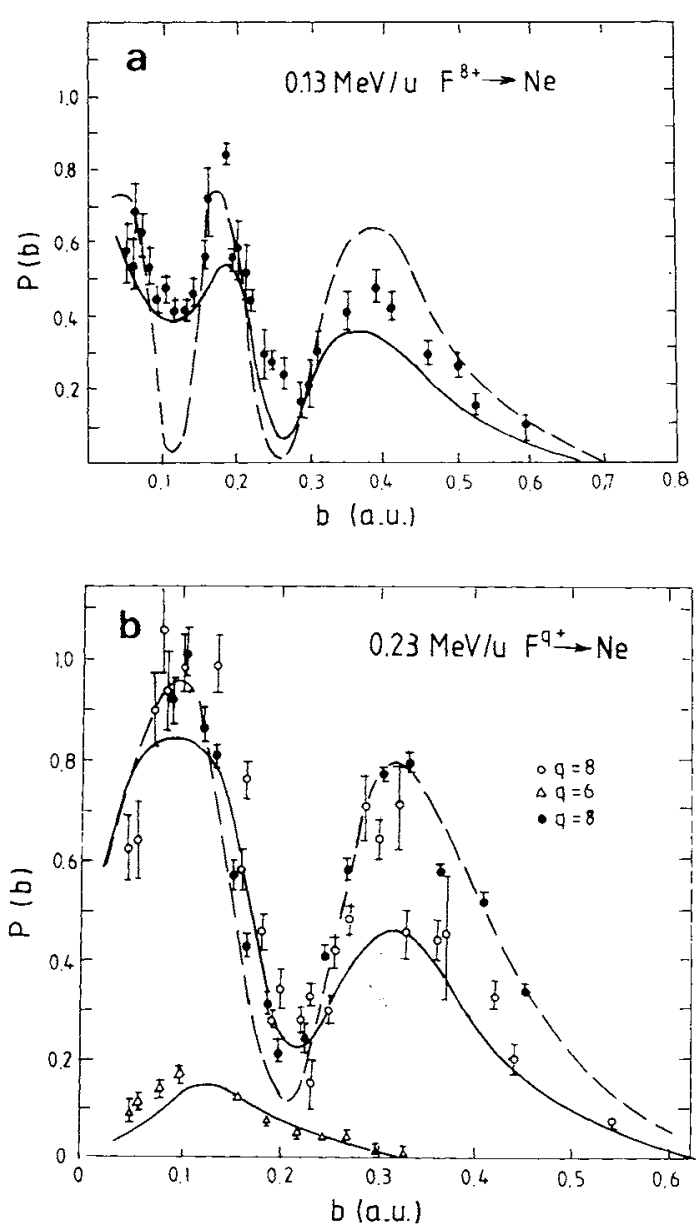

Fig. 2. $P(b)$ curves for the Ne $\mathrm{K}$ electron excitation for (a) 0.13 $\mathrm{MeV} / \mathrm{u}$ and (b) $0.23 \mathrm{MeV} / \mathrm{u} \mathrm{F}^{8+}-\mathrm{Ne}$ collision. Experimental values: ref. [1]; dashed line: ref. [3]; full line: this work. In the lower left the $P(b)$ curve for Ne K electron excitation is given for the system $\mathrm{F}^{6+}-\mathrm{Ne}$.

are already complicated many-electron systems although with small $Z$ which means that a non-relativistic calculation should be sufficient. But this relativistic version which we use here directly allows us to proceed to heavier systems where the relativistic effects become stronger or even dominant.

In fig. 1 we present the correlation diagram of the system $\mathrm{F}^{8+}-\mathrm{Ne}$ where the lowest level can be attributed to the F 1s state and the second to $\mathrm{Ne} 1 \mathrm{~s}$. This interchange is due to the high ionization of the fluorine. Because we are interested in the creation of holes in the Ne 1s shell we choose only those levels which are directly connected with this level or which are very near it. Thus the minimum number of levels which have to be taken into account as channels in the coupled channel calculations are the $1(1 / 2)$, $3(1 / 2), 4(1 / 2)$ and $1(3 / 2)$ levels. With this selection the number of one-particle channels in eq. (3) is 8 because each level can carry two electrons with an angular momentum projection on the internuclear axis + or - . As initial condition we know that there is only one electron in the two $1(1 / 2)$ states and that both $3(1 / 2)$ states are occupied. Although we also know the initial occupation of the higher levels in $\mathrm{F}$ and $\mathrm{Ne}$ at infinity we do not know the exact occupation of the $4(1 / 2)$ and $1(3 / 2)$ levels because electrons are transferred in the incoming part of the trajectory at large internuclear distances into these levels via dynamic couplings from levels which are not included in the 8-level calculation. To take these couplings into account in a pragmatic way we assumed that the $4(1 / 2)$ and $1(3 / 2)$ states are initially occupied with some number of electrons. We use this number in the calculations as a parameter which allows us to improve the agreement with the experimental results.

As result of the coupled channel calculations we get the amplitudes $c_{k n}$ from eq. (3) for all $n=1, \ldots$, $N$ where $N$ is the number of electrons and $k=1, \ldots$, $M$ with $M$ the number of channels. The agreement between the experimental $P(b)$ curves and our results in fig. 2 is good. The initial number of electrons in the four upper channels in our calculation are used as a parameter in order to get optimal agreement with the experiment. The numbers of electrons which we find are three and two for the two energies in increasing order. This result sounds very plausible from a physical point of view because for the higher energies the electrons in the higher levels will probably be more and more ionized during the collision.

For the case $\mathrm{F}^{6+}-\mathrm{Ne}$ the lowest two levels in the correlation diagram are in normal order again due to the stronger shielding of the fluorine nucleus. But both levels are filled with two electrons each in the experiment. Thus we perform the calculations for $0.23 \mathrm{MeV} / \mathrm{u}$ with the two electrons which we have learned are needed from the calculations discussed above, plus one electron which comes from the F $2 \mathrm{~s}$ shell. The result of this calculation is the dashed line in the lower left of fig. 2b. Again we find a good 
agreement with the experimental results. In order to understand also the filling of the $4(1 / 2)$ and $1(3 /$ 2 ) levels from the incoming $\mathrm{Ne}$ and/or $\mathrm{F}$ levels we have to increase in future calculations the number of states in the coupled channel calculations, so that the electron transfer between the outer levels can be taken into account.

As final statement we can say that the calculations presented here lead to a very physical interpretation: The levels which are used here are very realistic molecular states where a transfer between the lowest levels is sufficient to explain the hole production. This shows that the early simple models (like the Landau-Zehner model [7] in connection with the FanoLichten correlation diagrams [8]) in principle are also able to reproduce complicated many-particle collision systems as long as sophisticated ab initio self-consistent field wavefunctions and matrix elements together with a many-particle interpretation are used.

This work has been supported by Gesellschaft für Schwerionenforschung (GSI) Darmstadt and Deutsche Forschungsgemeinschaft.

\section{References}

[1] S. Hagmann, S. Kelbsch, H. Schmidt-Böcking, C.L. Cocke, P. Richard, S. Schuch, A. Skutlartz, J. Ullrich, B. Johnson, M. Meron, K. Jones, D. Trautmann and F. Roesel, Phys. Rev. A 36 (1987) 2603, and references therein.

[2] U. Wille, Excitation in ion-atom collisions, in: Coherence in atomic collision physics, eds. H. Beyer, K. Blum and R. Hippler (Plenum, New York, 1988) pp. 223-282, and references therein.

[3] W. Fritsch and C.D. Lin, Phys. Rev. A 31 (1985) 1166.

[4] A. Töpfer, A. Henne, H.J. Lüdde, M. Horbatsch and R.M. Dreizler, Phys. Lett. A 126 (1987) 11.

[5] W.-D. Sepp, D. Kolb, W. Sengler, H. Hartung and B. Fricke, Phys. Rev. A 33 (1986) 3679.

[6] J.F. Reading, Phys. Rev. A 8 (1973) 3262;

J. Reinhardt, B. Müller, W. Greiner and G. Soff, Phys. Rev. Lett. 43 (1979) 1307;

J.F. Reading and A.L. Ford, Phys. Rev. A 21 (1980) 124; H.J. Lüdde and R. Dreizler, J. Phys. B 16 (1983) 3973; 18 (1985) 107.

[7] L. Landau, Phys. Z. Sowjetunion 2 (1932) 40; C. Zehner, Proc. R. Soc. A 137 (1932) 696.

[8] U. Fano and W. Lichten, Phys. Rev. Lett. 14 (1965) 627; W. Lichten, Phys. Rev. 164 (1967) 131. 